\title{
ON A THEOREM OF BAKER, LAWRENCE AND ZORZITTO
}

\author{
L. SZÉKELYHIDI
}

\begin{abstract}
The result of J. Baker, J. Lawrence and F. Zorzitto on the stability of the equation $f(x+y)=f(x) f(y)$ is generalized by proving the following theorem: if $G$ is a semigroup and $V$ is a right invariant linear space of complex valued functions on $G$, and if $f, m$ are complex valued functions on $G$ for which the function $x \rightarrow f(x y)-f(x) m(y)$ belongs to $V$ for every $y$ in $G$, then either $f$ is in $V$ or $m$ is exponential.
\end{abstract}

In [1] J. Baker, J. Lawrence and F. Zorzitto, solving a problem of E. Lukacs on the stability of the functional equation $f(x+y)=f(x) f(y)$ proved that if $f$ is a function from a vector space to the real numbers satisfying

$$
|f(x+y)-f(x) f(y)|<\delta
$$

then $f$ is either bounded or exponential. This result was also generalized and simplified in [2]. Here we generalize this result in another way.

Let $G$ be a semigroup and $V$ be a linear space of complex valued functions on $G$. Then $V$ is called right invariant if $f$ belongs to $V$ implies that the function $x \rightarrow f(x y)$ belongs to $V$ for every $y$ in $G$. Similarly, we can define left invariant linear spaces, and we call $V$ invariant if it is right and left invariant.

The complex valued function $m: G \rightarrow C$ (C denotes the set of complex numbers) is called an exponential if for every $x, y$ in $G$ we have

$$
m(x y)=m(x) m(y) \text {. }
$$

Our main result is the following

TheOREM. Let $G$ be a semigroup and $V$ be a right invariant linear space of complex valued functions on $G$. Let $f, m: G \rightarrow C$ be complex valued functions for which the function $x \rightarrow f(x y)-f(x) m(y)$ belongs to $V$ for every $y$ in $G$. Then either $f$ is in $V$ or $m$ is an exponential.

Proof. Suppose that $m$ is not an exponential. Then there exist $y, z$ in $G$ with the property $m(y z)-m(y) m(z) \neq 0$. On the other hand we have, for all $x$ in $G$,

and hence

$$
\begin{aligned}
f(x y z)-f(x y) m(z)= & f(x y z)-f(x) m(y z)]-m(z)[f(x y)-f(x) m(y)] \\
& +f(x)[m(y z)-m(y) m(z)]
\end{aligned}
$$

$$
\begin{aligned}
f(x)= & (f(x y z)-f(x y) m(z))-(f(x y z)-f(x) m(y z)) \\
& +m(z)(f(x y)-m(y) f(x))] \cdot[m(y z)-m(y) m(z)]^{-1}
\end{aligned}
$$

Received by the editors November 26, 1980.

AMS (MOS) subject classifications (1970). Primary 39A15; Secondary 34D05.

Key words and phrases. Functional equation, stability. 
Here the right-hand side as a function of $x$ belongs to $V$, and hence so does $f$.

COROLlary. Let $G$ be a semigroup with identity and $V$ be an invariant linear space of complex valued functions on $G$. Let $f, m: G \rightarrow \mathbf{C}$ be complex valued functions for which the functions $x \rightarrow f(x y)-f(x) m(y)$ and $y \rightarrow f(x y)-f(x) m(y)$ belong to $V$ for every $y$ in $G$ and $x$ in $G$, respectively. Then either $f$ is in $V$ or $m$ is an exponential and $f=f(1) m$.

Proof. Suppose that $f$ is not in $V$. Then by the preceding theorem, $m$ is an exponential. On the other hand, the function $y \rightarrow f(y)-f(1) m(y)$ is in $V$ and for $x, y$ in $G$ we have

$$
\begin{aligned}
f(x y)-f(1) m(x y) & =f(x y)-f(x) m(y)+f(x) m(y)-f(1) m(x) m(y) \\
& =f(x y)-f(x) m(y)+[f(x)-f(1) m(x)] m(y) .
\end{aligned}
$$

If there is an $x_{0}$ in $G$ for which $f\left(x_{0}\right) \neq f(1) m\left(x_{0}\right)$, then $m$ belongs to $V$ and so does $f$, which is a contradiction. Hence $f=f(1) m$ which was to be proved.

REMARK. Here we make clear how the corollary generalizes the cited result. Let $G$ be an Abelian semigroup with identity and $V$ be the space of bounded complex valued functions on $G$. If $f, m: G \rightarrow C$ are functions for which there exist $M_{1}, M_{2}$ : $G \rightarrow[0,+\infty)$ such that

$$
|f(x y)-f(x) m(y)| \leqslant \min \left(M_{1}(x), M_{2}(y)\right)
$$

for all $x, y$ in $G$ then either $f$ is bounded or $m$ is exponential and $f=f(1) m$.

EXAMPLE. Let $G$ be a commutative topological group and $f: G \rightarrow \mathbf{C}$ be such that $x \rightarrow f(x+y)-f(x) f(y)$ is continuous for each $y$ in $G$. Then either $f$ is continuous or exponential. Other interesting examples can be constructed by taking $V$ to be the class of measurable or integrable functions on appropriate groups.

\section{REFERENCES}

1. J. Baker, J. Lawrence and F. Zorzitto, The stability of the equation $f(x+y)=f(x) f(y)$, Proc. Amer. Math. Soc. 74 (1979), 242-246.

2. J. Baker, The stability of the cosine equation, Proc. Amer. Math. Soc. 80 (1980), 411-416.

Department of Mathematics, Kossuth Lajos University, 4010 Debrecen, Hungary 